\title{
Formación ética como objetivo transversal de la escuela*
}

DOI: https://doi.org/10.18046/recs.i34.4225

\author{
Ethical Training as a School's Cross-Cutting Goal \\ Rodrigo Lagos-Vargas ${ }^{* *}$ \\ Universidad de Los Lagos (Osorno, Chile) \\ Claudia Keupuchur-Natalini ${ }^{* * *}$ \\ Universidad de Los Lagos (Osorno, Chile)
}

Marcelo Ramírez-Durán ${ }^{* * * *}$

Blas Pascal School (Osorno, Chile)

\footnotetext{
* Este trabajo forma parte de la línea de investigación del investigador principal, así como de una tesis de posgrado de la Maestría en Ciencias de la Educación de la Universidad de Los Lagos (Chile) desarrollada entre 2018 y 2019. Financiado con recursos propios. Artículo de revisión recibido el 15.08.2020 y aceptado el 26.01.2021.

** Académico del Departamento de Educación, Universidad de Los Lagos (Chile). Máster en Iniciación a la Investigación en Filosofía, Universidad Autónoma de Barcelona (España). Profesor de Filosofía de la Universidad de Concepción (Chile). Correo electrónico: rodlagos@ulagos.cl ORCID: https://orcid.org/oooo-ooo2-2254-7774

*** Estudiante tesista de la Maestría en Ciencias de la Educación, Universidad de Los Lagos (Chile). Profesora de Filosofía de la Universidad de Concepción (Chile). Correo electrónico: cnatalini@yahoo.com ORCID: https://orcid. org/oooo-0oo2-9446-2438

**** Profesor de inglés y traductor. Magíster en Desarrollo Curricular y Proyectos Educativos, Universidad Andrés Bello (Chile). Correo electrónico: marcelo.ramirez.duran@gmail.com ORCID: https://orcid.org/oooo-ooo3-4749-1778
} 


\section{Cómo citar/How to cite}

Lagos-Vargas, Rodrigo; Keupuchur-Natalini, Claudia; Ramírez-Durán, Marcelo (2021). Formación ética como objetivo transversal de la escuela. Revista CS, 34, 189-210.

https://doi.org/10.18046/recs.i34.4225 


\section{Resumen}

Este artículo analiza los resultados de investigaciones que exploran cómo abordamos la solución de dilemas morales, todo en aras de comprender sus repercusiones en la comprensión de la formación ética y moral, asimismo sus aportes a la educación transversal en el sistema educativo. Dicho análisis se nutre de investigaciones como la ICCS-2016, que describe aspectos preocupantes relacionados con la justificación de las dictaduras, la desconfianza en la justicia del Estado y la falta de empatía frente al fracaso de los demás. Esta indagación nos lleva a reconocer los aportes de la filosofía moral, la psicología moral y las neurociencias para entender la manera más adecuada de implementar la educación transversal y la formación ética-moral, esta vez desde los ámbitos emocional y racional como ineludibles. Esto resulta interesante dada la situación de sociedades como la chilena, que están en proceso de repensar el fundamento democrático legado por la otrora dictadura cívico-militar.

\section{PALABRAS CLAVE:}

formación ética, juicio moral, educación moral, educación transversal

This article analyzes the results of research carried out to explore how do we approach the solution to moral dilemmas, to understand its repercussions on the understanding of ethical and moral training, and its contributions to education of soft-skills in the education system. Nurtured by research such as ICCS - 2016, this study describes concerns related to the justification of dictatorships, mistrust in state justice, and the lack of sympathy for the failure of others. This inquiry leads us to acknowledge the contributions of moral philosophy, moral psychology, and neuroscience, to learn about the most appropriate way to implement transversal-skills education, as well as ethical and moral training, nevertheless, this time from the emotional and rational point of view. This is an interesting approach given the situation of societies such as Chile, which are currently rethinking the democratic foundations left by the former civic-military dictatorship.

\section{KEYWORDS:}

Ethical training, Moral Judgment, Moral Education, Transversal-Skills Education 



\title{
De la esperanza democrática ingenua a la necesidad de replantearla
}

\author{
"Regalé mis ojos para que la gente despierte". \\ (Gustavo Gatica, víctima cegada por la violencia \\ policial el 9 de noviembre de 2019)
}

A fines del siglo XX, cuando en América Latina acontecían importantes procesos sociopolíticos que ponían fin a las dictaduras cívico-militares y se anunciaba el advenimiento de sistemas democráticos, toda una generación de ciudadanos y actores sociales se mostraba más o menos optimista al vislumbrar ciertas posibilidades de transformación de la sociedad y de los valores que hasta entonces se proyectaban desde el Estado a la comunidad (Garretón, 1991; Sottoli, 200o). Las expectativas se orientaban a abordar la profunda desigualdad y pobreza a la que miles estaban condenados en nuestras sociedades. Una de las razones fundamentales se encontraba en la implementación de un sistema socioeconómico que tendía a la concentración de la riqueza; depredaba y privatizaba para el beneficio particular recursos naturales estratégicos; precarizaba y mal pagaba a muchos trabajadores.

La primera década del siglo XXI nos enseñó que aquella transformación ético-política que se esperaba en la nueva fase democrática latinoamericana, se transformó en un proceso de ajuste de las políticas de Estado para el mantenimiento del sistema socioeconómico, que se complacía con los resultados macroeconómicos.

De esta manera, el cambio fundamental que se produjo fue el despliegue de una estrategia de disciplina que, desde luego, no alteró el fundamento ético-político de las nuevas democracias, ni favoreció el fortalecimiento de las organizaciones sociales; más bien propició un incremento del acceso al consumo mediante el crédito, sin la educación financiera necesaria y con una oferta crediticia desmedida.

En este contexto, la búsqueda de satisfacción de anhelos y necesidades de bienes de consumo inmediatos o más duraderos (incluida la educación), que la generación anterior no pudo resolver o satisfacer, causó un endeudamiento que se constituyó en una estructura disciplinadora. De este modo, el dulce consumo y el amargo endeudamiento se transformaron en un dispositivo de control que obliga a mantener un flujo de pagos (en ocasiones abusivo o cercano a la usura), de tal suerte que se configura una forma de subjetividad representada por el trinomio crédito-consumo-endeudamiento. Una de las representaciones paradigmáticas de lo anterior la encontramos en el texto Chile actual, anatomía de un mito de Tomas Moulian, en el que se designa dicha configuración de esta subjetividad como la del ciudadano credit-card: 
Este ciudadano credit-card es normalizado, «puesto en orden», regulado por el consumo con pago diferido. Tiene que subordinar sus estrategias de conflicto, a sus estrategias de sobrevivencia como asalariado. Ha aprendido que su futuro está en seguir siendo un trabajador creíble. Esa credibilidad, vinculada a la sumisión, es la que le abre la puerta de futuros consumos ascendentes: el televisor-color, el automóvil, la casa propia. El crédito es un formidable factor de disciplinamiento, más eficiente en cuanto es plenamente mercantil, su mecanismo básico no es extraeconómico. (Moulian, 1997: 102)

De esta manera, la promesa de transformación del proyecto ético-político a través de la recuperación de la democracia en Latinoamérica resultó insuficiente en países como Chile. Esta situación comenzó a generar manifestaciones de aquellos grupos sociales que, precisamente, estaban menos disciplinados en el sistema de consumo del ciudadano credit-card. Dichos grupos sociales, que experimentaban los efectos disciplinadores de este modelo en sus familias, percibían una suerte de condena que se reproduciría en el futuro.

Durante el año 2006 comenzó en Chile la movilización estudiantil secundaria más grande hasta esa fecha, también denominada revolución pingüina. A partir de este hito, las movilizaciones sociales enfrentan y denuncian la incongruencia de esta versión de una democracia, empeñada en proyectarse como alternativa renovada del modelo impuesto por la dictadura, basada en una constitución de origen dictatorial que propicia la desigualdad e inequidad (Agacino, 2013; Ruiz, 2006). Este modelo establecía, por ejemplo, la primacía de una educación como espacio para el lucro, financiada por la disponibilidad de recursos económicos de las familias - que en un alto porcentaje las conducía al endeudamiento - a cambio de la obtención de resultados académicos y oportunidades de acceso a instituciones de educación superior. De este modo, el sistema educativo chileno no proveía oportunidades equivalentes (entre los establecimientos privados y públicos), capaces de promover el talento o la meritocracia; más bien, reproducía las condiciones económicas y socioculturales de los estudiantes y sus familias. Lo anterior se evidenciaba en los resultados de las evaluaciones estandarizadas nacionales o internacionales, aplicadas al sistema educativo.

A esta primera fase de movilizaciones seguirían otras, que incrementarían el cuestionamiento de las bases ético-políticas del modelo y sus consecuencias más indeseables, tales como la segregación, inequidad y el lucro desmedido (Mayol; Azócar, 2011) y, desde luego, el daño medioambiental.

Se puede afirmar que todo lo anterior no solo refleja un cuestionamiento a aspectos económico-políticos del desarrollo de un país como Chile o de Latinoamérica; más allá, expresa un malestar sociocultural más profundo que incluye las bases éticas de nuestras relaciones políticas: estas requieren ser objeto de reflexión, confrontación y disputa en aras de evitar que sigan reproduciéndose en un statu quo 
que se impone como destino, o bien se justifica en planteamientos decimonónicos que subestiman nuestra memoria histórica.

Este malestar, que se ha incubado en el despliegue de los proyectos democráticos contemporáneos latinoamericanos, debe reorientarse como una disputa hacia valores y principios que se desplegaron e instalaron a partir de unos espacios de poder, que les terminaron representando y proyectando a través de una estrategia biopolítica negativa (Mühle, 2010). Esto es una reducción de la vida por parte del poder, que la restringe y coacciona en sus potencialidades emergentes de la subjetividad; no obstante, comienzan a generar resistencias. Estas surgen no solo como anomalías eventuales, sino como síntomas estructurales que pueden llevarnos a desarrollar resistencias y cuestionamientos, los cuales posibilitarán la aparición de prácticas ético-políticas diferentes.

En la última década (2010-2020) hemos vivido en Chile una serie de episodios que refuerzan el sentido de un malestar que nos impulsa a pretender y exigir la reconfiguración de las bases de la convivencia ético-política de nuestra comunidad. Algunos de estos episodios han sido las colusiones empresariales de productos básicos, que quitan el velo de la ilusión del libre mercado; el financiamiento ilegal de la política, que cuestiona la independencia de los representantes para anteponer el bien común a intereses corporativos; el encubrimiento de la Iglesia Católica a sacerdotes pederastas y su desprotección a las víctimas; la corrupción de instituciones de seguridad como la policía uniformada, al malversar fondos públicos en beneficio de unos cuantos; y los montajes racistas en contra de miembros de las comunidades indígenas, por nombrar solo algunos de los casos más mediáticos, que tuvieron su efecto en el denominado estallido social del 18 de octubre del 2019.

La principal consecuencia del estallido y su relación con todo lo anterior se expresa en la definición de un referendo para la elaboración de una nueva constitución que, según los principales actores sociales que lo apoyan, debería replantear las bases de un nuevo acuerdo para la convivencia social y de un nuevo pacto ético-político, esta vez con un sentido que nos proyecte hacia una democracia que impulse la vida de todos y nos aleje, definitivamente, de un reformismo constitucional con trazas de la dictadura cívico-militar y de un sistema neoliberal feroz.

\section{Implicaciones de la educación cívica y ciudadana en la formación ética: consecuencias y reflexión}

El año 2018, la Asociación Internacional para la Evaluación del Logro Educativo publicó el último informe del Estudio Internacional de Educación Cívica y Formación Ciudadana (ICCS) aplicado el año 2016 (en Colombia, México, República Dominica- 
na y Chile), en el cual da cuenta de los aprendizajes de los estudiantes de $8 .^{\circ}$ grado en educación cívica y formación ciudadana. Este estudio analiza los conocimientos para comprender y valorar la vida en sociedad, así como nuestra forma de organizar la vida democrática, la capacidad de reflexionar sobre las instituciones, acciones y procesos desarrollados en las comunidades, y la habilidad para desarrollar y justificar opiniones y perspectivas respecto de estas. Asimismo, analiza las actitudes favorables acerca de la vida en democracia y la disposición a participar de forma efectiva en las comunidades. Si bien el estudio en cuestión se enfoca en el análisis del conocimiento de la cultura cívica democrática, hay algunas áreas interesantes respecto de su relación con la Formación Ética.

El estudio revela que ante la pregunta de si las dictaduras están justificadas cuando traen orden y seguridad o beneficios económicos, el promedio de aceptación obtenido en los países que participaron es de $69 \%$ y $65 \%$, respectivamente, para ambas consultas. Chile, por su parte, obtiene un $57 \%$ y 52 \% (Schulz; Ainley; Cox; Friedman, 2018: 29). Esta tendencia a la aceptación o justificación de las dictaduras demuestra cuán profunda es la inserción de valores que, lejos de expresar desconocimiento respecto de una cultura democrática, terminan validando su capacidad y validez como sistema político para asegurar aspectos fundamentales de la vida de las comunidades latinoamericanas, tales como seguridad o acceso a recursos.

Es igualmente interesante, desde la perspectiva de la formación ética, la manera como el estudio ICCS del 2016 analiza el acuerdo de los estudiantes con afirmaciones sobre la paz y la violencia (Schulz et al., 2018: 42) tales como "si las autoridades del país fracasan en actuar en aplicar las leyes y sancionar crímenes, los ciudadanos deberían organizarse para castigarles". En este caso, en la muestra de estudiantes de Chile un $65 \%$ de los participantes consideran que los ciudadanos deberían actuar, mientras que el promedio de los demás países participantes es de un $58 \%$ (Colombia ostenta el más bajo: $41 \%$ ). Esta diferencia de 7 puntos con respecto al promedio es la más alta respecto de los demás países y reflejaría, nuevamente, la desconfianza en la capacidad de la democracia actual para garantizar la justicia y la paz (seguridad), hecho que coincide con las consignas de las movilizaciones sociales que recriminan justamente al Estado. Asimismo, esto se relaciona con el ítem "la paz solamente se logra a través del diálogo y la negociación": para el caso de Chile, el porcentaje obtenido baja de un $80 \%$, obtenido en 2009, a un $68 \%$ en el estudio del 2016, a tal punto que queda en los niveles más bajos respecto de los demás países (Colombia alcanza el 80 \%).

Otro aspecto relevante del estudio ICCS, y de interés para este escrito, corresponde a los resultados en la categoría "sentido de empatía de los estudiantes con sus compañeros" (Schulz et al, 2018: 48), compuesto por 11 ítems, donde Chile obtiene 
como promedio total un resultado más bajo respecto de los demás participantes del estudio: $48 \%$. En 10 de los 11 ítems de la categoría, los resultados más bajos se obtienen en empatía respecto de si un compañero saca malas calificaciones; Chile obtiene un $42 \%$ frente a Republica Dominicana (que obtuvo el mayor porcentaje, $74 \%$ ) o Colombia (52\%), países que obtuvieron porcentajes más elevados. Cabe preguntarse en este punto si la empatía se explica en función de la instalación de valores vinculados a la meritocracia individual y la competencia, y el éxito académico se explica solo por el esfuerzo individual, sin consideración del contexto y el trabajo en equipo.

A partir de lo anterior cabe conocer cuál es el lugar en el sistema escolar chileno donde se recoge la posibilidad de la formación ética: esta yace en lo prescrito en los Objetivos Fundamentales Transversales (OFT) (Ministerio de Educación, 1996) y en las nuevas bases curriculares, a través de los denominados Objetivos de Aprendizaje Transversales (OAT). La manera como se comprende la implementación de esta enseñanza transversal parte de su incorporación en

(...) todos los sectores de aprendizaje y en todos los espacios de desarrollo curricular que componen la institucionalidad y la cultura escolar: las asignaturas, las prácticas de enseñanza y de aprendizaje en el aula, el clima organizacional y de relaciones humanas, las actividades recreativas, de libre elección, el Consejo de Curso, y las actividades definidas por los jóvenes, las actividades especiales periódicas, el sistema de la disciplina escolar, el ejemplo cotidiano, entre otros. (Ministerio de Educación, 2020)

La precisión de cómo conseguir una formación integral en la Educación chilena se ha declarado en instrumentos como el Plan de Formación Ciudadana, que desde el año 2016 tiene el objetivo de garantizar el desarrollo de una cultura democrática y ética en la escuela (Ministerio de Educación, 2016). Igualmente, a partir del año 2020 se ha incorporado al currículum nacional la asignatura de Ciencias para la Ciudadanía, con la pretensión de fortalecer los esfuerzos específicos que se realizan en asignaturas como Filosofía, Orientación, Historia y Geografía y Ciencias Naturales (Ministerio de Educación, 2019), que explicitan algún objetivo de aprendizaje relacionado con la formación ética.

No son muchas las investigaciones disponibles respecto de cómo se ha implementado esta educación transversal en Chile, pero algunas pueden ilustrar aspectos interesantes que ayudan a vislumbrar cómo se ha abordado la formación ética y cuáles han sido sus resultados. En González, Meza y Castro (2019) se analizaron las teorías subjetivas de los docentes de $7 .^{\circ}$ y $8 .^{\circ}$ grados respecto de la implementación de los objetivos transversales. La investigación se realizó con docentes de una población de alta vulnerabilidad. Sus principales conclusiones señalan que los 
docentes tienen dos focos principales en la educación transversal: la formación valórica y la convivencia escolar, ambos muy relacionados con la formación ética de los estudiantes. También se expresa que el quehacer docente se conduce, más que en prácticas planificadas y sistemáticas, en prácticas espontáneas y contingentes, dependientes de las emergencias contextuales intra o extraescolares que afectan de forma directa el proceso de enseñanza o la convivencia en la escuela. Desde luego, esto expresaría una debilidad en la manera de focalizar e implementar la educación transversal, pues la definición del éxito se entiende en la medida que este proceso sea coherente, persistente e intencionado. La priorización debería darse con acciones en que la escuela pueda instalarse como instancia privilegiada para fortalecer contextos intraescolares que contribuyan a la mejora de las debilidades de los contextos extraescolares, que muchas veces no pueden ser transformados. Es igualmente relevante que se hayan considerado factores emocionales y relacionales en la práctica formativa de la transversalidad. No obstante, resulta preocupante que los investigadores señalen cierta persistencia de los docentes a inducir emociones negativas para enfrentar las tensiones intra o extraescolares. Esto último genera la idea de que la transversalidad es una reacción que se enfoca en implementar acciones formativas correctivas para casos individuales, que poseen una alta carga emocional.

Desde luego, la comprensión de la formación ética en el sistema escolar requiere plantearse la modificación de la actual manera de implementar la transversalidad, orientándola desde una práctica contingente, fragmentada, pertinente solo para reestablecer la convivencia intraescolar que prosiga con la enseñanza; debe migrar hacia una práctica más sistemática, integrada en la comunidad escolar, persistente, que genera escenarios de experiencias positivas, emocionales, capaces de potenciar una convivencia y valores fundamentales para la comunidad escolar y coherentes con una sociedad democrática.

Es relevante, por otro lado, el hecho de que los profesores valoren la educación transversal y consideren que su labor ya no consiste solo en enseñar una disciplina particular (Cuadra; Salgado; Lería; Menares, 2018; Retuert; Castro, 2017; Saavedra, 2017). Sin embargo, reconocen que su implementación es parcial y se ve desfavorecida en un contexto donde solo se prioriza el éxito académico, fundamentalmente cognitivo, y en el que se da más relevancia a los resultados que se pueden evidenciar a partir de una calificación o puntaje (Saavedra, 2017).

Las investigaciones en el contexto de la formación ética en Chile, y en concreto, con estudiantes del sistema escolar, son casi inexistentes y eminentemente descriptivas. A pesar de esto, es posible determinar aspectos valiosos y, a la vez, preocupantes relacionados con el poco impacto que tendría la implementación de los OAT, en especial en el fortalecimiento de la reflexión ética de los estudiantes 
y en su desarrollo. Un ejemplo de esto yace en la competencia moral asociada a la resolución de dilemas morales de la vida en comunidad que repercuten en el fortalecimiento (o no) de la vida democrática, pues dicha competencia se relaciona con nuestra capacidad de enfrentar conflictos y la manera de interactuar con los demás (Lind, 2006; 2007).

En este sentido, investigaciones como las desarrolladas por Lagos-Vargas y Keupuchur-Natalini (2019) permiten identificar que los resultados en el despliegue de los $\mathrm{OAT}$ - tales como la reflexión ética, asociada al desarrollo de la competencia moral en estudiantes de 16 a 18 años de edad-son discretos, al margen de que provengan de estudiantes de establecimientos privados o públicos. Esto último se evidenciaría en el desempeño homogéneo y de poco impacto al aplicar el Test de Juicio Moral (Lind, 2014). Aproximadamente el $80 \%$ de los estudiantes que participaron en el estudio obtuvieron niveles de desarrollo que oscilaron entre bajo y medio, y solo un $20 \%$ de ellos alcanzó los niveles alto y muy alto.

Las orientaciones sobre cómo abordar una educación transversal y, en especial, una formación ética, deberían fortalecer el proceso formativo de los profesores; habrían de focalizarse, ante todo, en desarrollar una ética profesional, comprendida como parte de una habilidad reflexiva y crítica, que pueda ser compartida con los estudiantes para alcanzar el aprendizaje integral que se requiere (Silva; Ayala, 2020). Es relevante en esta instancia el apoyo y gestión del Ministerio de Educación y las instituciones educativas en el propósito de lograr el currículum escolar establecido. Para ello, deberá comprenderse qué aspectos resultan relevantes para conducir un proceso de formación ética y cómo estos posibilitan, por ejemplo, el desarrollo de la competencia moral de los estudiantes. En la misma línea, se deberá comprender cómo enfocar la educación transversal y la formación ética para la transformación de nuestras escuelas y sociedades en ámbitos capaces de fortalecer la democracia. Esto último es relevante para explorar ciertos elementos teóricos que ayuden a reconocer estos aspectos, porque lo realizado hasta la fecha resultaría insuficiente e ineficaz.

\section{Aspectos teóricos para una Formación ética en una sociedad democrática}

Los resultados del ICCS 2016 y sus implicancias en la formación ética de los estudiantes del sistema escolar chileno, que fueron analizados en los párrafos anteriores, dejan entrever uno de los problemas más relevantes del enfoque de la educación transversal actual: su alta priorización de los resultados académicos-cognitivos como criterio de calidad y, con esto, su cariz reproductor de una sociedad neoliberal 
que exacerba la idea de la meritocracia y de competencia entre los sujetos. No es de extrañar, con esto, que el enfoque de la educación transversal, y por extensión, de la formación ética, se explique como una estrategia eminentemente cognitiva, orientada a enfrentar situaciones contingentes que alteren el normal desarrollo del proceso de enseñanza (López, 2015). Esto último dado que, fundamentalmente, "se comenzó a sostener que la meta final del proceso educativo es humanizar lo más posible a la persona mediante el conocimiento, la razón y la técnica" (Ahumada; Martín, 2018).

Lo anterior implica que una transformación del contexto de formación transversal tradicional demanda a la formación de profesores el comprometerse con:

la necesidad de que las instituciones formadoras traduzcan sus postulados e intenciones de tipo moral en proyectos con un diseño que involucre actividades curriculares con valor formativo intencionado y explícito (...). (Meza; Guerrero, 2016)

Lo anterior requiere reflexionar sobre la manera como concebimos la educación del ser humano. En el caso específico de la formación ética, esto implica comprenderla como una tarea en ejercicio de la cual debe reconocerse que, al enfrentar desafíos o decisiones morales para orientar nuestro curso de acción, no solo nos orientamos desde un ámbito racional o cognitivo; también lo hacemos desde un ámbito emocional. $\mathrm{Al}$ respecto, existen planteamientos teóricos de gran influencia en los enfoques educativos de la formación ética, que pueden considerarse parte del debate filosófico, representado principalmente por las ideas de Hume y Kant. El primero señala que cualquier intento por suprimir o reducir nuestros sentimientos, deseos o emociones para determinar o actuar de acuerdo con un valor o mediante una virtud moral, haría que la moral deje de ser una disciplina práctica capaz de influir en la regulación de nuestras vidas y acciones (Hume, 2006: 46). El mismo autor señala también un rol subsidiario de la razón que la despoja de su prioridad explicativa del acto moral:

La razón, al ser fría y desapasionada, no motiva la acción [moral] y solo dirige el impulso recibido del apetito o inclinación, mostrándonos los medios de alcanzar la felicidad o de evitar el sufrimiento. (Hume, 2006: 206)

Si bien la perspectiva de Hume es la más extendida en cuanto a la fundamentación de la acción moral, nos parece igualmente relevante considerar los planteamientos de Spinoza. Este señala que la potencia de actuar de un cuerpo se ve incrementada o disminuida por los afectos a los que nos vemos expuestos (Spinoza, 2011: III, def. III, 209-210); y que al actuar en nosotros como pasiones, que conectan nuestra inte- 
rioridad y exterioridad, aumentan o disminuyen nuestra potencia (Tejeda; Martínez; Lagos-Vargas, 2020). Así, la afectividad se sitúa en una posición no solo irreductible de la condición humana, sino como elemento central en su devenir, en un sentido que nos proyecta o restringe.

Por su parte, Kant, sostenía que la ética y el ejercicio moral son aspectos conducidos por la razón, y que los juicios que emitimos para ponderar los medios y fines involucrados en la definición de máximas de acción, son necesariamente tareas lideradas por la razón (Kant, 2005: 69). En este mismo sentido, el autor pensaba que el ejercicio pedagógico, en su labor necesaria de formación moral, debía hacer que los niños aprendieran a pensar para conducir su acción en la sociedad (Kant, 200o: 19). Esto le hacía afirmar lo siguiente sobre la naturaleza moral del ser humano:

¿El hombre es por naturaleza, moralmente, bueno o malo? Ninguna de las dos cosas, pues no es por naturaleza un ser moral; sólo lo será cuando eleve su razón a los conceptos del deber y de la ley. Entretanto, se puede decir que tiene en sí impulsos originarios para todos los vicios, pues tiene inclinaciones e instintos que le mueven a un lado, mientras que la razón le empuja al contrario. (Kant, 2000: 93)

Asimismo, Kant considera las emociones o sentimientos como partes de la animalidad humana, que mediante el proceso de ilustración se subordinan a la racionalidad y se reorientan hacia aquello que le es propio a la condición humana: el ser racional. Estos planteamientos filosóficos han sido muy influyentes en el desarrollo de nuestro pensamiento ético y moral; representan dos tradiciones de filosofía que han tenido influencia e impacto en educación.

Desde luego, la reflexión ética como expresión del esfuerzo por racionalizar nuestras reglas para actuar en sociedad, así como la investigación para comprender la naturaleza de nuestro comportamiento moral en tanto ejercicio de nuestras reglas de acción social, no se han detenido: desde inicios del siglo XXI, han cobrado un interés muy relevante de otras disciplinas. Algunas de estas incorporaciones disciplinarias en la reflexión e investigación del fundamento de estas tradiciones ético-morales han sido los programas de investigación en neurociencia de la ética (Roskies, 2002; Koenigs et al., 2007) y de psicología moral (Greene; Nystrom; Engell; Darley; Cohen, 2004; Lind, 2007). Ambas disciplinas han planteado una reflexión, de base experimental y empírica, que ha estimulado el debate y que está permitiendo trasformar el modo en que la formación ética puede implementarse en la escuela y la sociedad.

Los estudios realizados a partir de estos programas de investigación han abordado el tema de la ética planteándose, ante todo, una indagación que pone a prueba las intuiciones filosóficas de emotivistas y racionalistas (o sus variantes), esta vez 
a partir de experimentos que analizan el comportamiento (conductual o cerebral) de individuos ordinarios, al abordar desafíos morales que, de algún modo, buscan representar la diversidad de los seres humanos y los distintos desarrollos morales que podemos alcanzar.

Algunos de los estudios relevantes en este sentido son, por ejemplo, los realizados por Joshua Greene et al. (2004): analizan de manera empírica las teorías filosóficas racionalista y emotivista, investigando cómo influyen en la elaboración de un juicio moral de acuerdo con la distinción entre cognición y emoción. Los autores citados señalan que existiría una configuración de nuestro cerebro, a partir de la cual, al enfrentarnos a dilemas morales eminentemente utilitaristas, tendemos a elaborar juicios morales con mayor compromiso cognitivo (activando estructuras neuronales evolutivas más recientes); y ante dilemas no utilitaristas, los juicios morales que se elaboran tienden a un mayor compromiso de las estructuras neuronales vinculadas a procesos emocionales. Greene et al. (2004) sostienen que, si bien la distinción cognición/emoción es real, es más bien una cuestión de grados evolutivos; en este marco, afirman que las respuestas cognitivas son consecuencia de procesos de racionalización de las emociones que siempre están en el trasfondo de las actividades cerebrales. Se establece así una primacía de la explicación emocional por sobre la racional, a fin de dar cuenta de los procesos de elaboración de los juicios morales que deberían afectar nuestra reflexión sobre el fundamento de la ética.

Por otro lado tenemos la psicología experimental, desarrollada a través de investigaciones como la de Cushman, Young y Hauser (2006): estos autores realizan una contrastación empírica entre las teorías ético-morales relativas al origen de los juicios morales desde perspectivas kantianas que expresan la idea de un proceso de elaboración racional consciente; $y$ desde perspectivas cercanas al intuicionismo de Moore (véase Lariguet, 2017), quien sostiene que los juicios morales surgen de intuiciones fundadas en conocimientos no expresables o inconscientes. El estudio concluye que al solicitar la justificación de juicios morales elaborados a partir de unos dilemas fundados en los principios de acción, intención y contacto ${ }^{1}$, los juicios morales de los individuos pueden explicarse mediante dos sistemas de producción: en uno emergen justificaciones morales disponibles para la reflexión consciente, que permitiría - mas no garantizaría - el papel del razonamiento consciente; y en el otro, la justificación se caracteriza por la aplicación de intuiciones que no están disponibles

1. Principio de acción: el perjuicio causado por la acción es moralmente peor que el perjuicio equivalente causado por omisión. Principio de intención: el perjuicio que se causa por medio de una acción intencional es moralmente peor que el perjuicio equivalente causado como efecto secundario no intencional. Principio de contacto: usar contacto físico para causar perjuicio a un individuo es moralmente peor que causarle un perjuicio equivalente a una víctima sin usar contacto físico (traducción propia de Cushman et al., 2006). 
para la discusión consciente y, más bien, permanecen implícitas. En definitiva, estos autores apuestan por un modelo multisistémico de elaboración de juicios morales y consideran erradas las propuestas clásicas como las de Kohlberg, quien asumía que todo juicio moral era fruto de la aplicación de un razonamiento consciente. En esta línea, existe una corriente pragmática de la psicología experimental representada por autores como Krebs y Denton (2005): señalan que el fundamento de los juicios morales se encontraría en aspectos cognitivos y afectivos, pero fundamentalmente con la preeminencia de procesos sociales como la cooperación.

Por su parte, estudios como el de Koenigs et al. (2007) contribuyen con un análisis neurobiológico, el cual busca determinar la contribución de ciertas estructuras cerebrales en la elaboración de los juicios morales y sus consecuencias analíticas. De modo concreto, su estudio compara a sujetos con daño en la corteza prefrontal ventromedial (CPVM) ${ }^{2}$ con sujetos normales. En este estudio se analizan sus respuestas a dilemas morales con y sin compromiso personal, esto es, con dilemas capaces de involucrarles solo en un cálculo racional o, por el contrario, en uno emocional. Los hallazgos reportaron que, ante dilemas de alto compromiso personal, los sujetos con daño CPVM los abordan significativamente desde una perspectiva utilitarista, a diferencia de los sujetos del grupo de control. Esto último sugeriría que ciertas estructuras neuronales del cerebro, como la corteza prefrontal ventromedial, resultan fundamentales para el funcionamiento del sistema intuitivo-afectivo en la respuesta a dilemas morales de fuerte contenido personal, no así en la respuesta a dilemas impersonales que comprometen el sistema consciente-racional del cerebro. Esto ratificaría la existencia de dos sistemas que permiten responder a dilemas morales - afectivo y racional-, los cuales interactúan y no son susceptibles de reducirse a solo uno de ellos sin afectar las estructuras propias del cerebro.

Una de las aplicaciones más relevantes de estos dos sistemas desde la psicología moral a la educación ha sido desarrollada por el psicólogo Georg Lind, mediante la elaboración de un instrumento que analiza los niveles de desarrollo de la competencia moral de los individuos y el método Konstanz de discusión de dilemas morales, el cual se funda en dos principios didácticos. El autor citado afirma que:

(...) cuando se enseña moral y competencias morales, (...) los maestros deben pensar qué clase de tareas y desafíos morales enfrentan los estudiantes en su vida cotidiana, de tal modo, que confronten a los pequeños con estas tareas morales que los ayuden a desarrollar estas competencias. (Lind, 2005)

2. Sujetos que conservan las capacidades de inteligencia general, razonamiento lógico y conocimiento declarativo de las normas sociales y morales, pero presentan compromiso de la respuesta emocional de carácter social. 
En el fondo teórico de este método de enseñanza se plantea que la respuesta moral se produce en virtud del despliegue de una competencia moral fundamentada en un sistema dual de respuesta emotivo-racional, que no es susceptible de reducción y solo es distinguible de manera formal. Esta perspectiva ha permitido a Lind diseñar e implementar tal propuesta para la enseñanza y reflexión ética, con el objetivo de favorecer el desarrollo de la competencia moral de los estudiantes. Esto se relaciona con la capacidad de enfrentar problemas o conflictos sobre la base de principios morales asumidos a través de la deliberación y la discusión, en lugar del ejercicio de la violencia o el engaño, teniendo en mente sociedades con una perspectiva ético-política relacionada con ideas democráticas (Lind, 2011). La ventaja del método de Lind radica en que es un programa estructurado de formación para monitores que pueden aplicarlo en contextos diversos. Del mismo modo, al contar con un instrumento de medición que permite evaluar los resultados de la implementación, algunas investigaciones que han aplicado el método señalan la buena percepción de los participantes y cierto nivel de mejora en el desarrollo de la competencia moral en grupos piloto. No obstante, se señala la necesidad de contar con un contexto propicio para su aplicación (véanse Serodio et al., 2016; Lerkiatbundit et al., 2006; Reinicke, 2015; Lajčiaková, 2016).

Con lo dicho, las investigaciones contemporáneas respecto de cómo abordamos la ética y la respuesta moral nos permiten señalar que necesariamente están involucrados en ello aspectos emocionales y racionales. Si bien existe aún discusión respecto de la eventual primacía de alguno de estos últimos, también existe cierto consenso en que ambos son relevantes y necesarios. Ahora bien, las implicaciones de lo anterior para la educación y la reflexión ética son relevantes, y aproximaremos algunas de ellas en la última sección del presente escrito.

\section{Conclusiones: formación ética desde la integralidad del ser humano}

El desarrollo de una formación ética planificada y sistemática, que integre la emoción y la razón en el proceso educativo, debe ser más evidente de lo que hoy es. Los resultados del ICCS o los ejemplos de estudios sobre educación transversal, o respecto del desarrollo de la competencia moral en estudiantes del sistema escolar, evidencian que, a pesar de su explicitud formal en ciertas normativas o directrices curriculares del sistema educativo, no hay razones para pensar que el sistema escolar contribuye de modo significativo al logro de los objetivos planteados; de momento, solo parecen intenciones de bajo impacto. 
Con lo anterior podemos afirmar que el sistema escolar no está en condiciones de abordar el desafío de una educación integral, más aún porque la formación de profesores y la gestión de la enseñanza en la escuela se centran de modo particular en el logro de resultados académicos en pruebas estandarizadas (nacionales o internacionales), más que en considerar la implementación de una educación con enfoque integral. Podríamos señalar, así, que se evidencia una priorización del logro individual, más que relevar la contribución a la comunidad.

De alguna manera, la insatisfacción ante el sistema escolar y, desde luego, ante el sistema ético-político, ha tenido su cenit en las movilizaciones del estallido social del 18 de octubre del 2019 en Chile: este expresa el descontento ante el actual modelo, la manera como se propició el desarrollo de nuestra democracia y, en efecto, de la educación, al no considerarse aspectos relevantes para el fortalecimiento de una sociedad con pretensiones democráticas más profundas.

Frente a lo anterior, el reto que se presenta yace en idear e implementar estrategias para robustecer y concretar el objetivo de una educación integral ética, que se orienten a potenciar la formación de la dimensión cognitiva de los estudiantes-que solo aborda la formación de la dimensión emocional en virtud de acontecimientos disruptivos o por alteraciones en el proceso educativo-, así como a equilibrar y complementar la formación cognitiva y emocional. No es posible cometer el error de creer que las emociones pueden ser sometidas o reducidas en nuestra manera de ser; por ello, es necesario discutir las bases de la educación transversal y su relación con el proyecto ético-político que vamos a apoyar desde nuestras comunidades. Esto último se ha tornado emergente en Chile, pues nos encontramos en el contexto excepcional de una inevitable discusión constituyente a partir de la cual, y dentro de otros muchos temas, la educación constituye una responsabilidad del Estado, más que solo de las familias. De este modo, el Estado debería gestionar e implementar una educación capaz de potenciar a los estudiantes plenamente sin establecer una prioridad a solo uno de los aspectos de la condición humana, como ha ocurrido hasta el momento.

Uno de los principales desafíos en la implementación de una educación transversal para potenciar la reflexión ética será focalizar los planes de estudio en la escuela, en virtud de resultados de aprendizaje que tengan en consideración las dimensiones actitudinales-emocionales, cognitivas y procedimentales de la enseñanza y el aprendizaje. Esto implicaría diseñar experiencias formativas capaces de dar cabida a las fortalezas y desafíos de los propios territorios, tal como se señala en el método Konstanz - por ejemplo, en materias económico-medioambiental, político-comunitaria, u otras, a fin de dotar de sentido próximo y experiencial, en sentidos coercitivo y afirmativo, los desafíos éticos y morales que deberán enfrentarse - . Desde luego, 
la formación de profesores debe considerar para esto una relación más directa, explícita y concreta con la transversalidad, que permita diseñar y desarrollar procesos de enseñanza planificados, no solo centrados en la dimensión cognitiva, sino que sea capaz de reconocer e incorporar la reflexión ética como elemento capaz de potenciar el saber y la interacción de nuestros estudiantes con su entorno, no solo como un recurso disponible para normalizar las conductas disruptivas en la escuela.

Asimismo, ha de propenderse al desarrollo de una investigación educativa que permita analizar buenas prácticas y establecer necesidades respecto de cómo se implementa la educación transversal, y en especial la formación ética, como aspecto relevante para fortalecer los procesos de reflexión y proyección de una sociedad democrática como la que necesitamos; una en que la competencia por el éxito económico individual, la exclusión de algún sector de la población y la depredación de los recursos naturales - en suma, la precarización de la vida- se reconozcan en tanto peligros que nos restringen y pauperizan como comunidad. Para alcanzar este objetivo deberían recogerse los aportes de la investigación empírica en ética y moral, ante todo respecto de la manera como enfrentamos nuestros desafíos morales. Esto nos dará la oportunidad de confrontar y ampliar un debate respecto de nuestras intuiciones teóricas y prácticas sociales al analizar, por ejemplo, cómo confrontar la cultura sexista - machista y el racismo en nuestras sociedades contra el pueblo mapuche en Chile o la inmigración (Huebner; Hauser, 2011; Loewe, 2017).

La relevancia de este análisis se ha dirigido a reconocer el problema ético-político en que se encuentra inmersa la educación, y cómo esta se ve impedida para desarrollar una formación transversal relevante, a la que urge apoyar con procesos reflexivos y críticos, capaces de integrar todos los ámbitos del ser humano sin caer en la tentación reduccionista de corte naturalista que podrían hacernos creer en soluciones parciales respecto de la formación ética, tal como lo señala Gracia-Calandín (2019). Para dicho autor, lo más relevante de la investigación neurocientífica aplicada a la educación es que permite confrontar estrategias de desmoralización de esta última, integrando elementos que emergen de resultados de investigaciones empíricas y teóricas que permiten orientar de mejor manera el despliegue en la escuela y la sociedad de tareas como la de una formación ética capaz de dar cuenta de los desafíos morales que hoy nos son ineludibles y urgentes. 


\section{Referencias}

Agacino, Rafael (2013). Movilizaciones estudiantiles en Chile: Anticipando el futuro. Educação en Revista, 14(1), 7-20. https://doi.org/10.36311/2236-5192.2013.v14n1.3294

Ahumada, Miguel; Martín, Xus (2018). Educación moral en Sudamérica: Un sistema pedagógico de transversalidad. Revista Brasileira de Educação, 23, 1-19. http://dx.doi.org/10.159o/ S1413-24782018230029

Cuadra, Daniel; Salgado, Jorge; Lería, Francisco; Menares, Néstor (2018). Teorías subjetivas en docentes sobre el aprendizaje y desarrollo socioemocional: Un estudio de caso. Revista Educación, 42(2), 1-20. https://doi.org/10.15517/revedu.v42i2.25659

Cushman, Fiery; Young, Liane; Hauser, Marc (2006). The Role of Conscious Reasoning and Intuition in Moral Judgment: Testing Three Principles of Harm. Psychological Science, 17(12), 1082-1089. https://doi.org/10.1111\%2Fj.1467-9280.2006.01834.X

Garretón, Manuel (1991). La democracia entre dos épocas: América Latina en 1990. Foro Internacional, 32(1[125]), 47-64. Recuperado de www.jstor.org/stable/27738402

González, Rodrigo; Meza, Omer; Castro, Pablo (2019). Teorías subjetivas de docentes sobre la implementación de los objetivos transversales en un contexto complejo. Psicoperspectivas, 18(2), 6-17. https://dx.doi.org/10.5027/psicoperspectivas-vol18-issue2-fulltext-1341

Gracia-Calandín, Javier (2019). Non-naturalistic Neuroethics for Moral Neuroeducation. En Moral Neuroeducation for a Democratic and Pluralistic Society (pp. 19-34), compilado por Patrici Calvo y Javier Gracia-Calandín. Springer International Publishing. https://doi. org/10.1007/978-3-030-22562-9

Greene, Joshua; Nystrom, Leigh; Engell, Andrew; Darley, John; Cohen, Jonathan (2004). The Neural Bases of Cognitive Conflict and Control in Moral Judgment. Neuron, 44, 389-400. Recuperado de https://www.sciencedirect.com/science/article/pii/So896627304006348

Huebner, Bryce; Hauser, Marc (2011). Moral judgments about altruistic self-sacrifice: When philosophical and folk intuitions clash. Philosophical Psychology, 24(1), 73-94. https://doi. org/10.1080/09515089.2010.534447

Hume, David (2014). Investigación sobre los principios de la moral. Madrid: Alianza Editorial.

Kant, Immanuel (200o). Sobre la pedagogía. Buenos Aires: El Aleph.

Kant, Immanuel (2005). Crítica de la Razón Práctica. México: FCE/UAM/UNAM.

Koenigs, Michael; Young, Liane; Adolphs, Ralph; Tranel, Daniel; Cushman, Fiery; Hauser, Marc; Damasio, Antonio (2007). Damage to the prefrontal cortex increases utilitarian moral judgements. Nature, 446, 908-911. https://doi.org/10.1038/natureo5631 
Krebs, Dennis y Denton, Kathy (2005). Toward a More Pragmatic Approach to Morality: A Critical Evaluation of Kohlberg's Model. Psychological Review, 112(3), 629-649. https://doi. org/10.1037/0033-295X.112.3.629

Lagos-Vargas, Rodrigo; Keupuchur-Natalini, Claudia (2019). Desarrollo de la competencia moral en estudiantes de secundaria: Una reflexión sobre la Formación Ética en la escuela. Opción, 35(90), 1301-1321. Recuperado el 22 de junio de 2020, de https://produccioncientificaluz. org/index.php/opcion/article/view/30498/31544

Lariguet, Guillermo (2017). Intuicionismo y razonamiento moral. Derecho PUCP, 79, 127-150. https://dx.doi.org/10.1880o/derechopucp.201702.007

Lajčiaková, Petra (2016). The effect of dilemma discussion on moral judgement competence in helping-professions students. European Journal of Science and Theology, 12(2), 7-18. Recuperado de http://www.ejst.tuiasi.ro/Files/57/2_Lajciakova.pdf

Lerkiatbundit, Sanguan; Utaipan, Parichat; Laohawiriyanon, Chonlada; Teo, Adisa (2006). Impact of the Konstanz method of dilemma discussion on moral judgment in allied health students: a randomized controlled study. Journal of Allied Health, 35(2), 101-108.

Lind, Georg (2005). Guía n. ${ }^{\circ}$ : el método Konstanz de dilemas morales. Trabajo presentado en Taller El método Konstanz de dilemas morales, Ministerio de Educación Nacional, Bogotá, Colombia. Recuperado de https://www.cacatu.do/wp-content/uploads/2019/o5/GuiaDesarrollo-Dilemas-Morales.pdf

Lind, Georg (2006). Effective moral education: The Konstanz method of dilemma discussion. Hellenic Journal of Psychology, 3, 189-196. Recuperado de https://www.researchgate.net/ publication/237446309_Effective_Moral_Education_The_Konstanz_Method_of_Dilemma_ Discussion

Lind, Georg (2007). La moral puede enseñarse: Manual teórico- práctico de la formación moraly democrática. Ciudad de México: Trillas.

Lind, Georg (2011). Promoviendo las competencias morales y democráticas: expresarse y escuchar a otros. Postconvencionales, 3, 26-41. Recuperado de http://190.169.94.12/ojs/ index.php/rev_post/article/view/6288\#.YJw_vOu23OR

Lind, Georg (2014). The moral judgment test. Recuperado de http://moralcompetence.net/ material/moral/messen/MCT-span9-2015\%20(c)\%2oLind.pdf

López, Carlos (2015). Evolución y desarrollo actual de los Temas Transversales: posibilidades y límites. Foro de Educación, 13(18), 143-16o. Recuperado de https://forodeeducacion.com/ ojs/index.php/fde/article/view/317

Loewe, Daniel (2017). Virtudes, racionalidad y el desarrollo moral. Alpha: revista de artes, letras $y$ filosofia, 44, 243-251. http://dx.doi.org/10.4067/So718-22012017000100243 
Mayol, Alberto; Azócar, Carla (2011). Politización del malestar, movilización social y transformación ideológica: el caso "Chile 2011". Polis. Revista Latinoamericana, 10(30), 163-184. http://dx.doi.org/10.4067/So718-65682011000300oo8

Meza, Marisa; Guerrero, Alex (2016). Competencia moral-democrática en futuros profesores de Pedagogía en educación básica en Chile ¿Importa la formación inicial docente? Perfiles educativos, 38(154), 41-56. https://doi.org/10.22201/iisue.24486167e.2016.154.57652

Decreto 40 de 2006 (3 de febrero). Establece objetivos fundamentales y contenidos mínimos obligatorios para la educación básica y fija normas generales para su aplicación. Biblioteca del Congreso Nacional. Recuperado de https://bit.ly/2NvzOPI

Ministerio de Educación (2016). Orientaciones para la elaboración del plan de formación ciudadana. Recuperado de https://media.mineduc.cl/wp-content/ uploads/sites/28/2016/o7/Orientaciones-para-la-elaboraci\%C3\%B3n-del-Plan-deFormaci\%C3\%B3n-Ciudadana.pdf

Ministerio de Educación (2019). Bases curriculares para la implementación en tercero y cuarto de Enseñanza Media. Recuperado de https://www.curriculumnacional.cl/614/ articles-133992_recurso_27.pdf

Ministerio de Educación (2020). ¿Qué es la transversalidad Educativa? Recuperado de https:// www.ayudamineduc.cl/ficha/que-es-la-transversalidad-educativa-5

Moulian, Tomás (1997). Chile actual. Santiago de Chile: Lom Ediciones.

Mühle, María (2010). Sobre la vitalidad del poder: una genealogía de la biopolítica a partir de Foucault y Canguilhem. En Michel Foucault: neoliberalismo y biopolitica (pp. 397-432), editado por Vanessa Lemm. Santiago de Chile: Ediciones Universidad Diego Portales.

Reinicke, Martina (2015). Inclusion as a Moral Challenge: the Potential of the Konstanz Method of Dilemma Discussion" (KMDD'). Filozofia Publiczna i Edukacja Demokratyczna, 4(1), 88101. http://dx.doi.org/10.14746/fped.2015.4.1.5

Retuert Gabriel; Castro, Pablo (2017). Teorías subjetivas de profesores acerca de su rol en la construcción de la convivencia escolar. Polis, 16(46), 321-345. https://dx.doi.org/10.4067/ So718-65682017000100321

Roskies, Adina (2002). Neuroethics for the new millenium. Neuron, 35(1), 21-23. https://doi. org/10.1016/So896-6273(02)00763-8

Saavedra, Priscilla (2017). Contextualización curricular de objetivos de aprendizaje transversales (OAT): Estudio de caso en un colegio particular pagado [tesis de maestría]. Recuperado de https://repositorio.uc.cl/handle/11534/22007

Schulz, Wolfram; Ainley, John; Cox, Cristián; Friedman, Tim (2018). Percepciones de los jóvenes acerca del gobierno, la convivencia pacifica y la diversidad en cinco países de América 
Latina: Estudio Internacional sobre Educación Cívica y Ciudadana 2016 de la IEA Informe Latinoamericano. Nueva Zelanda: Springer Nature.

Serodio, Aluisio; Kopelman, Benjamin; Bataglia, Patricia (2016). The promotion of medical students' moral development: a comparison between a traditional course on bioethics and a course complemented with the Konstanz method of dilemma discussion. International Journal of Ethics Education, 1(1), 81-89. https://doi.org/10.1007/s40889-016-0oo9-8

Silva, Rafael; Ayala, Ana (2020). Enseñanza de la ética profesional y su transversalidad en el currículo universitario. Cali: Editorial Universidad Icesi. https://doi.org/10.18046/EUI/ vc.3.2020

Sottoli, Susana (200o). La política social en América Latina bajo el signo de la economía de mercado y la democracia. Revista Mexicana De Sociología, 62(4), 43-65. https://doi. org/10.2307/3541152

Spinoza, Baruch (2011). Ética. Madrid: Alianza Editorial.

Tejeda, Cristian; Martínez, Rodrigo; Lagos-Vargas, Rodrigo (2020). Filosofía de la educación en Spinoza: acerca de la sumisión voluntaria y devenir racional. Inédito. 